\title{
Digital Risk Management for Data Attacks against State Evaluation
}

\author{
M. Nalini, Anvesh Chakram
}

\begin{abstract}
Understanding reasonable framework cyber attacks is essential for creating material assurance and recuperation measures. Propelled attacks follow exploited contact at diminished expenses and recognize capacity. This paper behaviors chance investigation of joined data trustworthiness and handiness attacks against the office framework state evaluation. We will in general contrast the consolidated attacks and unadulterated honesty attacks - false data infusion attacks. A safety record for defenselessness appraisal to those two sorts of attacks is arranged and created because a blended number connected science drawback. We will in general demonstrate that such joined attacks will prevail with less assets than false data infusion attacks. The consolidated attacks with confined data of the framework design also open gifts to keep camouflage against the undesirable data location. At last, the risk of joined attacks to dependable framework activity is assessed abuse the outcomes from defenselessness evaluation and attacks sway examination. The discoveries during this paper are substantial and upheld by $a$ top to bottom contextual investigation.
\end{abstract}

Keywords-Transmission line measurements, Transmission line matrix methods, power systems, Security, Indexes, State estimation, False data injection, Network topology.

\section{INTRODUCTION}

The continuously computerize framework present more information, subtleties, and manage during a instance span style than its non-arranged antecedents. The profiting uses of this advancement is State Evaluation, Terminal Units give measure data by means of Information and Communication Technology foundation like Supervisory administration and data Acquisition framework. The State Evaluation furnishes the administrator with partner degree gauge of the condition of the electrical network. This state data is then utilized and handled by the vitality the energy management system for best power flow, contingency analysis, and programmed age the executives. Security of give relies upon the energy management system, that progressively relies upon a dependable State Evaluation,. As referenced in the Supervisory administration and data Acquisition framework is helpless against a curiously large scope of security dangers. A group of trustworthiness information assault, alluded to as false data infusion assault, has been examined with sizable consideration. With adjusting the estimation data, this assault will pass the unfortunate data Detection among State Evaluation by interfering of Terminal Units, the

Revised Manuscript Received on July 13, 2019.

M.Nalini, Assistant Professor, Department of Computer Science and Engineering, Saveetha School of Engineering, Saveetha Institute of Medical and Technical Sciences, Chennai, India

Anvesh Chakram, UG Scholar, Department of Computer Science and Engineering, Saveetha School of Engineering, Saveetha Institute of Medical and Technical Sciences, Chennai, India databases and information technology programming framework inside the inside. Be that as it may, such false correspondence connects to the middle, or possibly the data detection assault needs escalated assault assets like the learning of the framework design and furthermore the capacity to degenerate the uprightness on a gathering of estimations. Denial-of-service assaults, a sort of availability assault, are a lot "less expensive" to achieve, especially if RTUs convey by means of uncertain correspondence channels. During this paper, we will in general target consolidated assaults any place the SE is debased by every uprightness assaults and openness assaults in the meantime. We think about joined assaults and FDI assaults underneath very surprising degrees of ill-disposed data and assets.

\section{RELATED WORK}

Our proposed system is intently connected with i) dispersed cross-layer utility boost hypothesis for electronic correspondence systems and ii) in-organize Computation methods. Inside the in-organize calculation writing, our work is most connected with, any place built up a system current design on behalf of the in-arrange calculation in gadget arrange applications. The system in expands the client flow protection law inside the system flow writing to in-arrange calculation applications. Notwithstanding, the design in is confined to easy tree topologies that are utilized for information conglomeration in gadget systems. In qualification, the system flow design works with conventional dag, That are the preeminent applicable designs for cutting edge distributed computing programming structures, similar to wood fairy and Map Reduce. Additionally, in our network design, we tend to consolidate common system usefulness and correspondence/calculation worth capacities, that weren't thought of in [3]

Our system design conjointly shares a few similitude's with the load shedding and appropriated asset the executive's downside of flow procedure systems. Anyway our work contrasts from these works inside the accompanying 2 fundamental angles: beginning, however the trouble of flow awkwardness in flow processing systems was conjointly known in the flow imbalance was brought about by very surprising flow creation and utilization rates among up flow and down flow nodes. In qualification, the flow unevenness during this work is due to sub-calculation in mists that could be a basically very surprising reason. Second the errand toserver task.

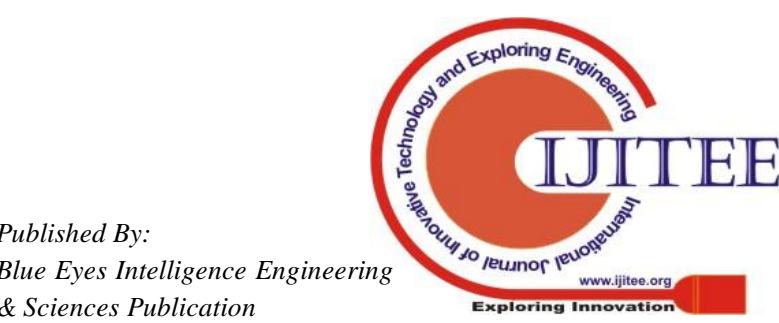


Association is expected to run and along these lines the creators solely studied the start to finish utility rate augmentation. In refinement, the errand to-cut off task relationship is moreover $50 \%$ of the streamlining in our task. Our system flow design conjointly has associations to the chart implanting issues in diagram hypothesis. anyway these issues differ from our own in that their implanting goals were to reduce various diagram theoretic execution measurements.

\section{EXISTING SYSTEM}

The problem of finding the communication and innetwork computation schedule of a given discretional perform of distributed knowledge therefore on maximize the rate of computation.

Capacitated communication network and a number of other infinite sequences of supply knowledge every of that is out there at some node within the network. A perform of the supply knowledge is to be computed within the network and made out there at a sink node that's additionally on the network. The schema to compute the perform is given as a directed acyclic graph (DAG).

We want to generate a computation and communication schedule within the network to maximize the speed of computation of the perform for discretional perform (represented by DAG).

\section{Drawbacks:}

-High computation time.

-Total migration time and service downtime.

\section{LITERATURE SURVEY}

1. The VIKING venture: An activity on versatile control of intensity systems, AnnaritaGiani ; Shankar Sastry ; Karl H. Johansson, 2009. This paper exhibits the work on flexible and secure power transmission and dissemination created inside the VIKING (indispensable foundation, systems, data and control framework the board) venture. VIKING gets subsidizing from the European Community's Seventh Framework Program. We will display the consortium, the inspiration driving this examination, the fundamental target of the undertaking together with the present status.

2. Hamed Mohsenian-Rad, 2013. False information infusion assaults are as of late presented as a class of digital assaults against keen matrix's observing frameworks. They plan to bargain the understanding of network sensors and pharos estimation units. Ongoing investigations have demonstrated that if the administrator utilizes the DC, the state evaluation to decide the present conditions of the power framework, the aggressor can alter the assault vector with the end goal that the assault stays undetected and effectively passes the ordinarily utilized buildup based terrible information identification tests. In any case, in this paper, we analyze the likelihood of actualizing a bogus information infusion assault when the administrator utilizes the more down to earth $\mathrm{AC}$, i.e., nonlinear, state estimation. We portray such assaults when the assailant has impeccable and flawed information of the present conditions of the framework. As far as we could possibly know, this is the primary paper to address fake information infusion assaults beside non-direct state evaluation.

3. Deepjyoti Deka ; Ross Baldick ; Sriram Vishwanath, 2015. Meter estimations in the power matrix are helpless to control by enemies that can prompt blunders in state evaluation. In this paper exhibits a universal structure to study assaults on state evaluation by enemies equipped for infusing awful information into evaluations and further, of sticking their gathering. Through these two methods, a novel 'perceptible sticking' assault is planned that changes the state evaluation in spite of flopping awful information identification checks. Contrasted with normally consider 'shrouded' information assaults, these assaults have lower costs and a more extensive plausible working locale. It is demonstrated that the whole space of sticking expenses can be partitioned into two locales, with particular diagram cut based definitions for the plan of the ideal assault. The most noteworthy understanding emerging from this outcome is that the ill-disposed capacity to stick evaluation s changes the ideal 'perceivable sticking' assault structure just if the sticking expense is not exactly a large portion of the expense of terrible information infusion. A polynomial time surmised calculation for assault vector development is created and its viability in assault configuration is exhibited through recreations on IEEE test frameworks.

4. Djordje Atanackovic; Greg Dwernychuk; Raju Vinnakota, 2010. State estimator application is the center propelled application in the Energy Management framework (EMS) that gives significant contributions to other propelled arrange applications that are executed to decide control framework security in the continuous. Those applications incorporate transient and voltage security investigation that are additionally in charge of computation and download of the therapeutic activity plans equipping examples to the field in the continuous. Therefore, state estimator execution quality is very imperative to BCTC ongoing activities. State estimator depends on the nature of status and simple constant telemetry and is additionally emphatically subject to the nature of system design parameters, for example, line and transformer impedances and charging permissions. The goal of this paper is to portray the upkeep practices embraced at British Columbia Transmission Corporation to guarantee high caliber and heartiness of EMS state estimator with an accentuation on system parameter quality following and improvement.

5. Jinping Hao ; Robert J. Piechocki ; Dritan Kaleshi ; Woon Hau Chin ; Zhong Fan, 2015.. This paper examines vindictive false information infusion assaults on the wide region evaluation and observing framework in keen networks. To begin with, strategies for building inadequate stealth assaults are created for two normal situations: 1) irregular assaults in which discretionary evaluations can be undermined; and 2) directed assaults in which indicated state factors are changed. It is as of now shown that stealth assaults can generally exist if the quantity of bargained evaluations surpasses a specific worth. In this paper, it is discovered that irregular imperceptible assaults can be

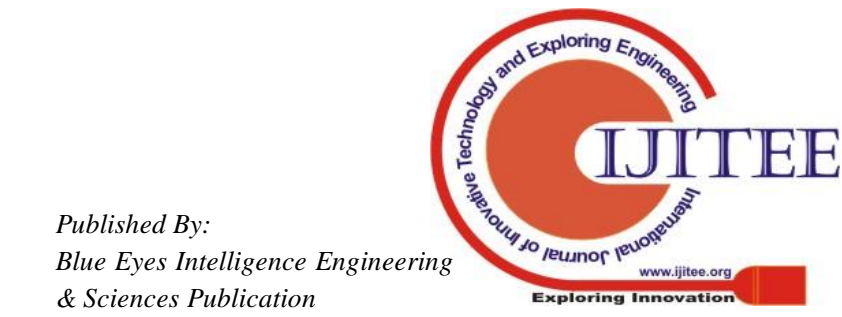


practiced by changing just an a lot more modest number of evaluations than this worth. It is outstanding that shielding the framework from noxious assaults can be accomplished by making a specific subset of evaluations invulnerable to assaults. A productive ravenous hunt calculation is then planned to rapidly observe this separation of evaluations to be secured to guard beside stealth assaults. It is demonstrated that this eager calculation has nearly a similar presentation as the savage power strategy, yet without the combinatorial intricacy. Third, a hearty assault discovery strategy is examined. The identification strategy is planned dependent on the hearty head part examination issue by presenting component astute requirements. This technique is demonstrated to have the option to recognize the genuine evaluations, just as assaults notwithstanding when just halfway perceptions are gathered. The recreations are led dependent on IEEE test frameworks.

6. State evaluation is one of the essential capacities in present day control lattice tasks that give administrators situational mindfulness and is utilized by a few applications like possibility investigation and power markets. A few explores in the ongoing history have featured the weakness of state estimators to quiet fake information infusion assaults that sidestep terrible information location systems. They essentially centered around recognizing stealthy assault vectors and portraying their effects on state gauges. Presented alleviation events either center around covering the impact of assaults through excess evaluations or anticipate assaults by expanding the digital security of related sensors and correspondence channels. The arrangements dependent on these disconnected methodologies create explicit suspicions about the idea of assaults and of the framework, which are frequently prohibitive and terribly insufficient to manage powerfully developing digital dangers and changing framework setups. We propose an online oddity location calculation that uses burden gauges, age plans, and synchrophasor information to identify evaluation oddities. We give some knowledge into the elements that influence the presentation of the proposed calculation. We likewise depict an observational technique to get the base assault extents and the location limits for gathering determined false positive and genuine positive rates. At long last, we assessed the exhibition of the proposed calculation utilizing the IEEE 14 transport power framework design for a few measures (false positive, false negative, and edges). We saw that the best execution of the proposed calculation depends on finding the correct harmony between the base assault greatness and discovery edges. We additionally seen that the base assault extents and identification limits could be additionally improved using a mix of increasingly exact figures and PMU evaluations.

7. Power frameworks are being presented to digital assaults because of the high joining of data innovation and the helplessness of correspondence systems. Obtainable false information assaults research center around dc state evaluation, we demonstrate that an aggressor can build an imperceptible assault vector against air conditioning state estimation dependent on a couple of estimations in the assaulting locale related with limit transports without knowing the full topology and parameter data of the whole power arrange. A cycle approach is received to get the assault vector. The reenactments on the transport and transport frameworks are utilized to exhibit the rightness and viability of the planned assault conspire. This article gives a premise to think about the assault practices below the air conditioner case, and a hypothetical manual for create insurance systems and recognition strategies.

8. An information confining assault is displayed to abuse the awful information discovery and recognizable proof components at a regular ISO/RTO direct focus. Specifically, the planned system assault outlines ordinary meters as wellsprings of terrible information and causes the manage focus to expel helpful evaluations from the encircled meters. The planned system assault utilizes subspace data of intensity framework evaluations; the system topology nor the system restrictions are necessary for building the assault It is demonstrated that the planned assault is fit for bothering the rule framework state gauge by a subjective degree utilizing just $60 \%$ of the basic evaluations. Ramifications of this assault on power framework tasks are talked about, and the assault execution is assessed utilizing benchmark frameworks.

\section{PROPOSED SYSTEM}

\section{Advantages}

- Collective attacks can be successful with less resources

- It reduces the total migration time and service downtime.

\section{MODULES}

\section{User Interface Design}

This is the essential design of our work. The indispensable job for the customer is to go login page to customer page. This design has made for the assurance reason. During the login page we must enter login customer id and parole. It will ensure customer name and parole is organize or not. On the ability that we will in universal enter any unacceptable customer name or parole. In general won't enter into login page to customer page it'll demonstrates mistake message. These lines we keep an eye on territory unit keeping from unapproved customer going in the login page to customer page. It'll offer a legit security for our task. Accordingly server contain customer id and parole server conjointly check the verification of the customer. It well improves the insurance and maintain from unaccepted customer goes into the system. In our scheme we watch out for zone unit exploitation Java Script Program for creating style. This design in general support the login customer and server verification. 


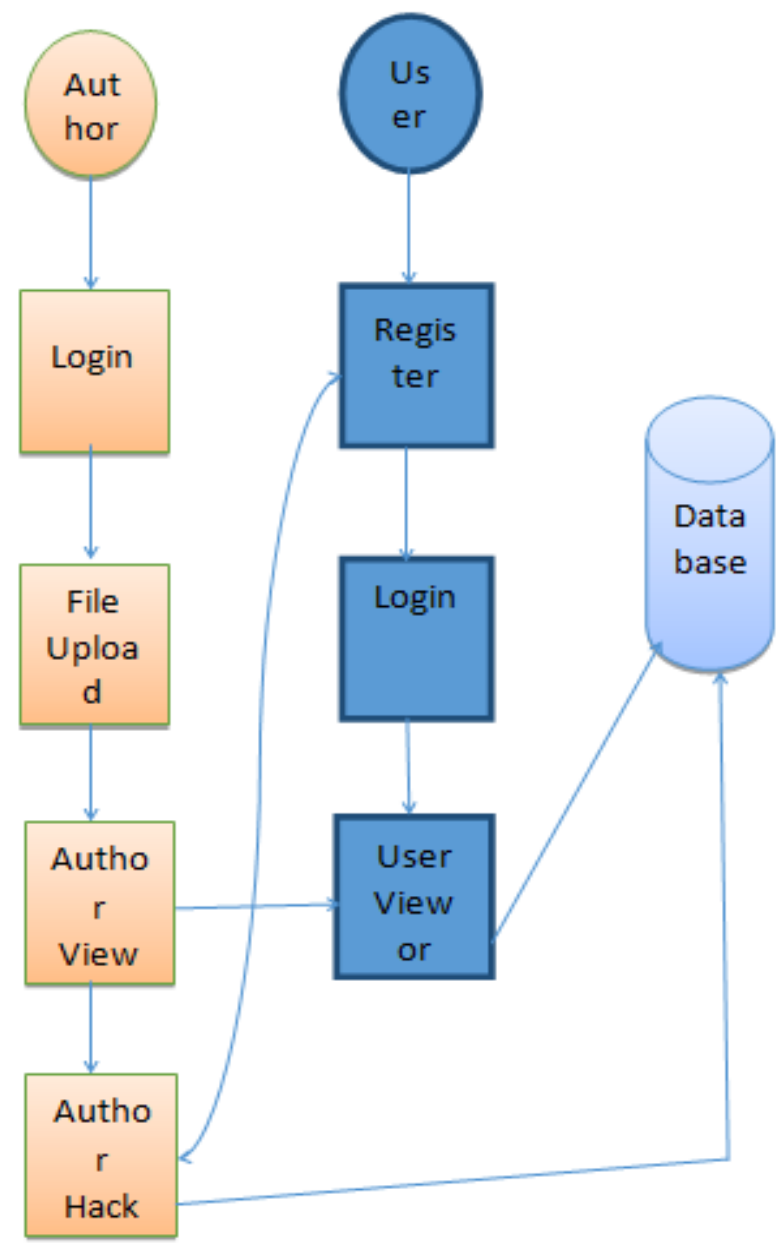

Fig. 1 System Architecture

\section{Author Login}

This is the second unit of our undertaking. The essential work for the creator is to progress login page to customer page. This module has created for the insurance reason, throughout this login page we should enter login customer id and information. It'll check customer name and information is coordinate or not (legitimate customer id and substantial secret key). On the off likelihood that we will in general enter any invalid customer name or information we will in general can't go into login page to customer page it'll indicates error message. These lines will in general square measure maintain from unaccepted customer getting in the login page to customer page. It'll offer a fair security for our task. Accordingly server contain customer id and information server conjointly check the authentication of the customer. It will improves the safety and maintain unaccepted customer exit into the page. In our undertaking we will in general square measure abuse java script program for creating style. In general we will approve the login customer and server authentication. If type any unacceptable customer name or information we will in general can't go into login page to customer page it will demonstrates error message. Therefore we will in general square measure maintain from unaccepted customer getting in the login page to customer page. They offer a genuine safety for our job. In this way server control customer id and information server conjointly check the confirmation of the customer. It well improves the insurance and maintain from unaccepted customer goes into the system. In our task we will in general square measure abuse java script program for creating style. We will in general accept the login customer and server verification.

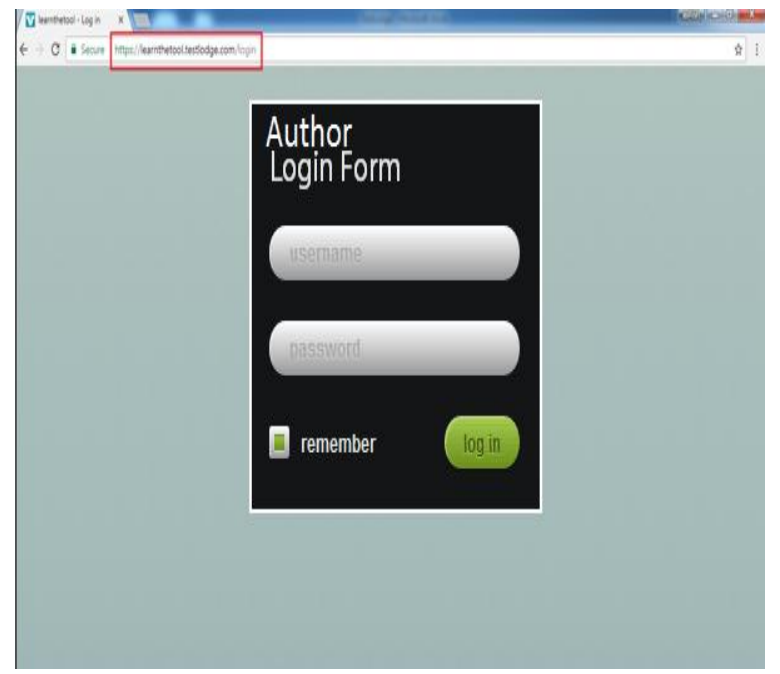

Fig. 2 Author Login Page

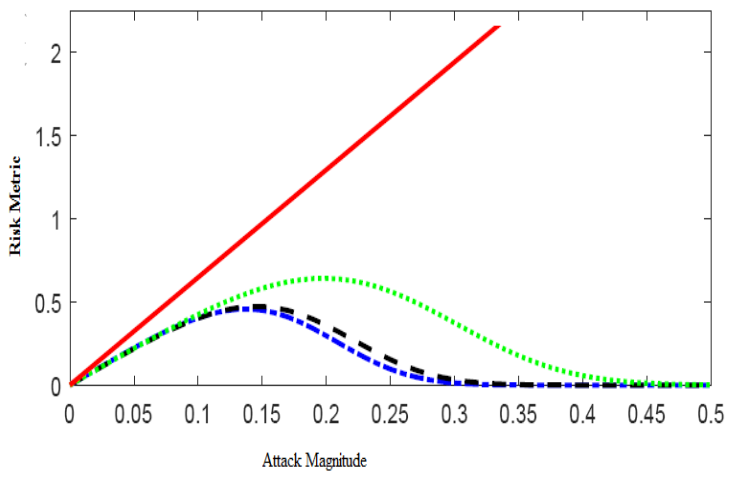

Fig. 3 Risk Metric

\section{Book Upload}

This is the Third module in our project, monthly Magazine transfer the web site and free transfer book and pdf customer access the Magazine or book used the free website the one in every of best website the Magazine several author list and book list.

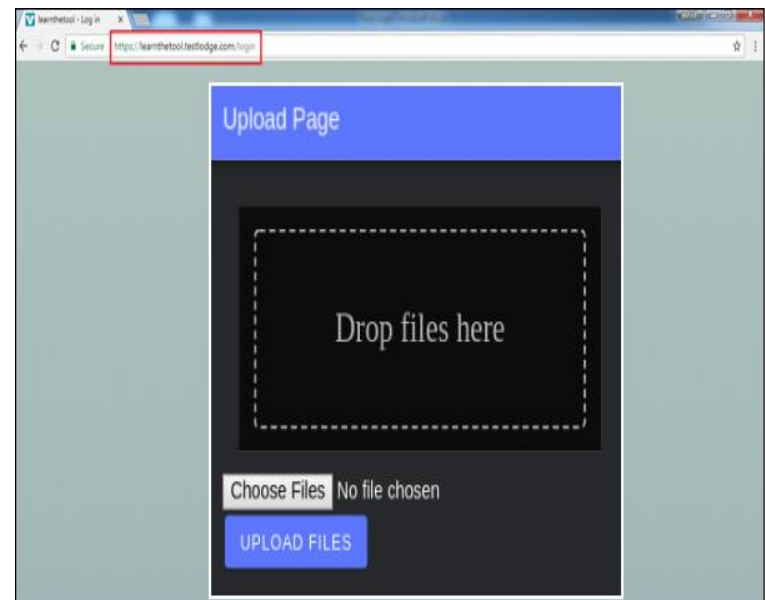

Fig. 4 Book Upload Page

Published By: Blue Eyes Intelligence Engineering \& Sciences Publication 


\section{CONCLUSION}

We have contemplated the matter of unequivocal the most rate of registering and correspondence elements of evaluations taken by hubs in a very identifier system to a picked sink hub. We've focused on isobilateral capacities, since they're a characteristic class of elements of enthusiasm for uniform indicator systems. There are assortment of headings for future work. To start with, the outcomes demonstrated inside the last area are for systems during which the quantity of synchronous transmissions is the restricting imperative. There are elective reflection configurations, similar to framework and line, in which a consistent throughput to the closest neighbors is conceivable, anyway the prohibitive issue is that calculation of the perform required should in any case need bound information to be transferred. Second, we have not contemplated non symmetric capacities, and neither would we be able to gain lower limits on feasible rate for all potential isobilateral capacities. Another characteristic expansion is to present joint disseminations on the indicator readings, and affirm limits on the basic rate of calculation of capacities. We will in general accept this is regularly a sensibly troublesome drawback. At long last, an information divinatory way to deal with the issue is wide open.

\section{REFERENCES}

1. Giani, S. Sastry, K. H. Johansson, and H. Sandberg, "The Viking project:an initiative on resilient control of power networks," in 2nd International Symposium on Resilient Control Systems, 2009, pp. $31-$ 35 .

2. Y. Liu, P. Ning, and M. K. Reiter, "False data injection attacks against state estimation in electric power grids," in Proc. of the 16th ACM Conf. on Computer and Comm. Security, New York, 2009, pp. 21-32.

3. W. Wang and Z. Lu, "Cyber security in the smart grid: Survey and challenges," Computer Networks, vol. 57, no. 5, pp. 1344-1371, 2013.

4. Nalini, M. and Uma Priyadarsini, To Improve the Performance of Wireless Networks for Resizing the Buffer, Proceedings of the 2019 international IEEE Conference on Innovations in Information and Communication $\quad$ Technology, $\quad$ Apr 2019. [DOI>10.1109/ICIICT1.2019.8741406]

5. D. Deka, R. Baldick, and S. Vishwanath, "Optimal data attacks on power grids: Leveraging detection measurement jamming," in Proc. of IEEE Int. Conf. Smart Grid Communications (SmartGridComm), Miami Florida, USA, Nov. 2015, pp. 392-397.

6. Nalini, M. and Anbu, S., "Anomaly Detection Via Eliminating Data Redundancy and Rectifying Data Error in Uncertain Data Streams", Published in International Journal of Applied Engineering Research (IJAER), Vol. 9, no. 24, 2014

7. R. S. Ross, "Nistsp - 800 - 30 rev 1: Guide for conducting risk assessments," NIST, techreport, Sep. 2012.

8. G. Hug and J. A. Giampapa, "Vulnerability assessment of AC state estimation with respect to false data injection cyber-attacks," IEEE Transactions on Smart Grid, vol. 3, no. 3, pp. 1362-1370, Sep. 2012.

9. Uma Priyadarsini and Nalini, M, Transient Factor- Mindful Video Affective Analysis- A Proposal for Internet Based Application, Proceedings of the 2019 international IEEE Conference on Innovations in Information and Communication Technology, Apr 2019. [DOI>10.1109/ICIICT1.2019.8741466]

10. H. Sandberg, A. Teixeira, and K. H. Johansson, "On security indices for state estimators in power networks," in First Workshop on Secure Control Systems (SCS), Stockholm, 2010.

11. A. Teixeira, K. C. Sou, H. Sandberg, and K. H. Johansson, "Secure control systems: A quantitative risk management approach," IEEE Control Systems, vol. 35, no. 1, pp. 24-45, 2015.

12. Nalini, M. and Anbu, S., "A Novel Framework for Automatic Data Maintenance for DBMS Development", Published in Australian Journal of Basic and Applied Sciences (AJBAS), Vol. 9, no. 36, pp.198-206, 2015.
13. A. Teixeira, G. D'an, H. Sandberg, and K. H. Johansson, "A cyber security study of a SCADA energy management system: Stealthy deception attacks on the state estimator," Proceedings of IFAC World Congress, Aug 2011.

14. O. Kosut, L. Jia, R. J. Thomas, and L. Tong, "Malicious data attacks on the smart grid," IEEE Transactions on Smart Grid, vol. 2, no. 4, pp. 645-658, 2011.

15. A. Ashok, M. Govindarasu, and V. Ajjarapu, "Online detection of stealthy false data injection attacks in power system state estimation,'IEEE Transactions on Smart Grid, vol. PP, no. 99, p. 1, 2016

16. O. Vukovic, K. C. Sou, G. Dan, and H. Sandberg, "Network-aware mitigation of data integrity attacks on power system state estimation,' IEEE Journal on Selected Areas in Communications, vol. 30, no. 6, pp.1108-1118, 2012.

17. K. Pan, A. M. H. Teixeira, M. Cvetkovic, and P. Palensky, "Combined data integrity and availability attacks on state estimation in cyberphysical power grids," in Proc. IEEE Int. Conf. Smart Grid Communications(SmartGridComm), Nov. 2016, pp. 271-277. 